\title{
Asymptotic symmetries and dynamics of three-dimensional flat supergravity
}

\author{
Glenn Barnich, ${ }^{a}$ Laura Donnay, ${ }^{a}$ Javier Matulich ${ }^{b, c}$ and Ricardo Troncoso ${ }^{b, d}$ \\ ${ }^{a}$ Physique Théorique et Mathématique Université Libre de Bruxelles, and \\ International Solvay Institutes Campus Plaine, \\ C.P. 231, B-1050 Bruxelles, Belgium. \\ ${ }^{b}$ Centro de Estudios Científicos (CECs), \\ Casilla 1469, Valdivia, Chile \\ ${ }^{c}$ Departamento de Fúsica, Universidad de Concepción, \\ Casilla, 160-C, Concepción, Chile \\ ${ }^{d}$ Universidad Andrés Bello, \\ Av. República 440, Santiago, Chile \\ E-mail: gbarnich@ulb.ac.be, ldonnay@ulb.ac.be, matulich@cecs.cl, \\ troncoso@cecs.cl
}

ABSTRACT: A consistent set of asymptotic conditions for the simplest supergravity theory without cosmological constant in three dimensions is proposed. The canonical generators associated to the asymptotic symmetries are shown to span a supersymmetric extension of the $\mathrm{BMS}_{3}$ algebra with an appropriate central charge. The energy is manifestly bounded from below with the ground state given by the null orbifold or Minkowski spacetime for periodic, respectively antiperiodic boundary conditions on the gravitino. These results are related to the corresponding ones in $\mathrm{AdS}_{3}$ supergravity by a suitable flat limit. The analysis is generalized to the case of minimal flat supergravity with additional parity odd terms for which the Poisson algebra of canonical generators form a representation of the super-BMS 3 algebra with an additional central charge.

Keywords: Conformal and W Symmetry, Space-Time Symmetries, Gauge-gravity correspondence, Classical Theories of Gravity

ArXiv ePrint: 1407.4275 


\section{Contents}

1 Introduction $\quad 1$

2 Minimal $\mathcal{N}=1$ flat supergravity in $3 \mathrm{D} \quad 2$

3 Asymptotic behaviour, canonical generators and super-BMS 3 algebra 4

4 Energy bounds and Killing spinors $\quad 6$

$\begin{array}{lll}4.1 & \text { Energy bounds from quantum superalgebra } & 6\end{array}$

4.2 Asymptotic Killing spinors $\quad 6$

$\begin{array}{lll}4.3 & \text { Exact Killing spinors of bosonic zero mode solutions } & 7\end{array}$

5 Flat limit of asymptotically $\mathrm{AdS}_{3}$ supergravity $\quad 8$

5.1 Asymptotic behaviour of minimal $\mathrm{AdS}_{3}$ supergravity, canonical generators $\begin{array}{lr}\text { and superconformal symmetry } & 8\end{array}$

$\begin{array}{ll}5.2 \text { Vanishing cosmological constant limit } & 10\end{array}$

6 Asymptotic structure of $\mathcal{N}=1$ "reloaded" flat supergravity 12

$\begin{array}{ll}\text { A Conventions } & 13\end{array}$

\section{Introduction}

When restricting the gravitational phase-space to conical spacetimes $[1,2]$ in 2+1-dimensional flat supergravity, it has been shown $[3,4]$ that one can define neither linear momentum nor supercharge but only energy and angular momentum because the asymptotic dynamics does not allow for the associated symmetries. The absence of unbroken supercharge in this context has important physical implications as it can serve as a mechanism to ensure vanishing cosmological constant for the vacuum while at the same time boson and fermion masses need no longer be degenerate [5].

The same kind of symmetry breaking occurs in pure Einstein gravity with negative cosmological constant for a suitably restricted phase-space, but disappears when consistently relaxing the boundary conditions in order to allow for a richer asymptotic structure [6]: in this case, the asymptotic symmetry group is enlarged and contains not only $\mathrm{SO}(2,2)$ but the conformal group in two dimensions. At the same time, the phase-space now includes, besides the angular defects, further "zero mode solutions", such as the BTZ black hole $[7,8]$ and more generally, two arbitrary functions that make up the coadjoint representation $[9,10]$ of two copies of the Virasoro algebra at central charge $c^{ \pm}=3 l / 2 G$ (see also [11-13] for more recent discussions and applications). The results on an enhanced 
asymptotic structure have been extended to $\mathrm{AdS}_{3}$ supergravity for which the boundary dynamics is governed by the superconformal algebra [14-16].

A similarly rich asymptotic structure for flat three-dimensional gravity can be defined at null infinity $[17-19]^{1}$ and is connected through a well-defined flat-space limit to the one of $\mathrm{AdS}_{3}$ [21-26]. In particular, the limit of BTZ black holes are cosmologies [27, 28] whose horizon entropy can be understood from symmetry arguments [29, 30] consistent with those of the $\mathrm{AdS}_{3}$ case [31], while the boundary dynamics [32] is a flat limit of Liouville theory [33], obtained through a Hamiltonian reduction from a chiral $\operatorname{ISO}(2,1)$ Wess-Zumino-Witten theory [34, 35].

The purpose of the present paper is to extend this asymptotic analysis to the simplest $\mathcal{N}=1$ flat supergravity in three dimensions. As expected from the AdS and the flat case in the absence of fermions [36], the reduced phase space with its Dirac bracket of charges turns out to coincide with the coadjoint representation of the centrally extended asymptotic symmetry algebra, viz. the super-BMS 3 algebra, which now includes the full Poincaré superalgebra as a subalgebra.

Note that in the context of Galilean conformal algebras, superalgebras isomorphic to the super-BMS $\mathrm{BM}_{3}$ algebra, but with a different physical interpretation for the generators, have been constructed previously [37, 38] by taking a non-relativistic limit of the superconformal algebra (see also [39, 40] for finite-dimensional versions).

In the next section, we briefly describe $\mathcal{N}=1$ flat supergravity in three dimensions together with its Chern-Simons formulation. Additional conventions are given in the appendix A.

The main part of the paper is section 3, where we provide suitable fall-off conditions and work out the asymptotic symmetry algebra, the general solution to the supergravity equations of motion consistent with the boundary conditions, the transformation laws of the functions parametrizing solution space and the Poisson bracket algebra of the canonical symmetry generators together with the associated central charge.

Finally, in section 4, we discuss energy bounds and the Killing spinor equation, while section 5 is devoted to rederiving the flat space results from the corresponding ones for asymptotically $\mathrm{AdS}_{3}$ supergravity by rephrasing the latter in a suitable gauge that allows one to perform the vanishing cosmological constant limit in a straightforward way. Section 6 is devoted to the minimal locally supersymmetric extension of the most general threedimensional gravity theory without cosmological constant that leads to first order field equations for the dreibein and the spin connection. Due to additional parity odd terms, the Poisson algebra of canonical generators is given again by the centrally extended super$\mathrm{BMS}_{3}$ algebra, but now with an additional central charge for the superrotation subalgebra.

\section{Minimal $\mathcal{N}=1$ flat supergravity in $3 \mathrm{D}$}

The minimal locally supersymmetric extension of General Relativity in three dimensions with $\mathcal{N}=1$ gravitino was constructed in [41-43]. Nowadays, it is well-known that the

\footnotetext{
${ }^{1}$ As shown in [20], these results can be naturally extended to the case of conformal gravity in three dimensions.
} 
theory can be described in terms of a Chern-Simons action in the cases of negative [44] or vanishing [45] cosmological constant. In the latter case, different extensions of the theory have been developed in e.g., [46-51].

Let us begin by considering the simplest case which corresponds to $\mathcal{N}=1$ supergravity theory with vanishing cosmological constant. The gauge field $A=A_{\mu} d x^{\mu}$ is given by

$$
A=e^{a} P_{a}+\omega^{a} J_{a}+\psi^{\alpha} Q_{\alpha},
$$

where $e^{a}, \omega^{a}$ and $\psi^{\alpha}$ stand for the dreibein, the dualized spin connection $\omega_{a}=\frac{1}{2} \epsilon_{a b c} \omega^{b c}$, and the (Majorana) gravitino, respectively; while the set $\left\{P_{a}, J_{a}, Q_{\alpha}\right\}$ spans the super-Poincaré algebra, given by

$$
\begin{aligned}
& {\left[J_{a}, J_{b}\right]=\epsilon_{a b c} J^{c} ; \quad\left[J_{a}, P_{b}\right]=\epsilon_{a b c} P^{c} ; \quad\left[P_{a}, P_{b}\right]=0,} \\
& {\left[J_{a}, Q_{\alpha}\right]=\frac{1}{2}\left(\Gamma_{a}\right)_{\alpha}^{\beta} Q_{\beta} ; \quad\left[P_{a}, Q_{\alpha}\right]=0 ; \quad\left\{Q_{\alpha}, Q_{\beta}\right\}=-\frac{1}{2}\left(C \Gamma^{a}\right)_{\alpha \beta} P_{a},}
\end{aligned}
$$

where $C$ is the charge conjugation matrix. The action then reads

$$
I[A]=\frac{k}{4 \pi} \int\left\langle A, d A+\frac{2}{3} A^{2}\right\rangle,
$$

where the bracket $\langle\cdot, \cdot\rangle$ stands for an invariant nondegenerate bilinear form, whose only nonvanishing components are given by

$$
\left\langle P_{a}, J_{b}\right\rangle=\eta_{a b}, \quad\left\langle Q_{\alpha}, Q_{\beta}\right\rangle=C_{\alpha \beta},
$$

and the level is related to the Newton constant according to $k=\frac{1}{4 G}$. Hence, up to a boundary term, the action reduces to

$$
I=\frac{k}{4 \pi} \int 2 R^{a} e_{a}-\bar{\psi} D \psi
$$

where $\bar{\psi}_{\alpha}=C_{\alpha \beta} \psi^{\beta}$ is the Majorana conjugate, while the curvature two-form and the covariant derivative of the gravitino are defined as

$$
R^{a}=d \omega^{a}+\frac{1}{2} \epsilon^{a b c} \omega_{b} \omega_{c} \quad ; \quad D \psi=d \psi+\frac{1}{2} \omega^{a} \Gamma_{a} \psi .
$$

By construction, the action is invariant, up to a surface term, under the local supersymmetry transformations spanned by $\delta A=d \lambda+[A, \lambda]$, with $\lambda=\epsilon^{\alpha} Q_{\alpha}$, whose components read

$$
\delta e^{a}=\frac{1}{2} \bar{\epsilon} \Gamma^{a} \psi ; \delta \omega^{a}=0 ; \delta \psi=D \epsilon .
$$

Analogously, the field equations $F=d A+A^{2}=0$, whose general solution is locally given by $A=G^{-1} d G$, reduce to

$$
R^{a}=0 ; T^{a}=-\frac{1}{4} \bar{\psi} \Gamma^{a} \psi ; D \psi=0,
$$

where $T^{a}=d e^{a}+\epsilon^{a b c} \omega_{b} e_{c}$ is the torsion two-form. 


\section{Asymptotic behaviour, canonical generators and super-BMS $\mathrm{B}_{3}$ algebra}

The aim is now to provide a suitable set of fall-off conditions for the gauge fields at infinity that (i) extends the one of the purely gravitational sector so as to include the bosonic solutions of interest, and (ii) is relaxed enough so as to enlarge the set of asymptotic symmetries from $\mathrm{BMS}_{3}$ to a minimal supersymmetric extension thereof. In order to fulfill these requirements, the behaviour of the gauge fields at the boundary is taken to be of the form

$$
A=h^{-1} a h+h^{-1} d h,
$$

where the radial dependence is completely captured by the group element $h=e^{-r P_{0}}$, and $^{2}$

$$
a=\left(\frac{\mathcal{M}}{2} d u+\frac{\mathcal{N}}{2} d \phi\right) P_{0}+d u P_{1}+\frac{\mathcal{M}}{2} d \phi J_{0}+d \phi J_{1}+\frac{\psi}{2^{1 / 4}} d \phi Q_{+},
$$

where the functions $\mathcal{M}, \mathcal{N}$, and the Grassmann-valued spinor component $\psi$ are assumed to depend on the remaining coordinates $u, \phi$.

The asymptotic symmetries correspond to the set of gauge transformations, $\delta A=$ $d \lambda+[A, \lambda]$, that preserves this behaviour. When applied to the dynamical gauge fields $A_{\phi}$, one finds that the Lie-algebra-valued parameter $\lambda$ must be of the form

$$
\lambda=\xi^{a}(u, \phi) P_{a}+\chi^{a}(u, \phi) J_{a}+2^{1 / 4} \epsilon^{+}(u, \phi) Q_{+}+2^{1 / 4} \epsilon^{-}(u, \phi) Q_{-},
$$

with

$$
\begin{aligned}
\xi^{0}(u, \phi) & =\frac{1}{2} \mathcal{N}(u, \phi) \chi^{1}(u, \phi)+\frac{1}{2} \mathcal{M}(u, \phi) \xi^{1}(u, \phi)-\xi^{1 \prime \prime}(u, \phi)+\frac{1}{2} \epsilon^{-}(u, \phi) \psi(u, \phi) \\
\xi^{2}(u, \phi) & =-\xi^{1 \prime}(u, \phi) \\
\chi^{0}(u, \phi) & =\frac{1}{2} \mathcal{M}(u, \phi) \chi^{1}(u, \phi)-\chi^{1 \prime \prime}(u, \phi) \\
\chi^{2}(u, \phi) & =-\chi^{1 \prime}(u, \phi) \\
\epsilon^{+}(u, \phi) & =\frac{1}{\sqrt{2}}\left(\chi^{1}(u, \phi) \psi(u, \phi)-2 \epsilon^{-\prime}(u, \phi)\right)
\end{aligned}
$$

in terms of functions $\chi^{1}, \xi^{1}, \epsilon^{-}$of $u, \phi$ and prime denotes a derivative with respect to $\phi$. When applied to the Lagrange multipliers $A_{u}, \lambda$ is restricted further to depend only on three arbitrary functions of the angular coordinate, two bosonic ones $Y(\phi), T(\phi)$, and one fermionic $\mathcal{E}(\phi)$,

$$
\chi^{1}(u, \phi)=Y(\phi) \quad, \quad \epsilon^{-}(u, \phi)=\mathcal{E}(\phi) \quad, \quad \xi^{1}(u, \phi)=T(\phi)+u Y^{\prime}(\phi),
$$

and, at the same time, the field equations are required to hold in the asymptotic region: the fields $\mathcal{M}, \mathcal{N}, \psi$ become subject to the conditions

$$
\partial_{u} \mathcal{M}=0 \quad, \quad \partial_{u} \mathcal{N}=\partial_{\phi} \mathcal{M} \quad, \quad \partial_{u} \psi=0,
$$

\footnotetext{
${ }^{2}$ Hereafter we assume light-cone coordinates in tangent space. See appendix A for the $\Gamma$-matrices representation and further conventions.
} 
which are trivially solved by

$$
\mathcal{M}=\mathcal{M}(\phi) \quad, \quad \mathcal{N}=\mathcal{J}(\phi)+u \mathcal{M}^{\prime}(\phi) \quad, \quad \psi=\Psi(\phi) .
$$

The phase space is thus reduced to three arbitrary functions of the angular coordinate, $\mathcal{M}$, $\mathcal{J}, \Psi$, whose transformation laws under the asymptotic symmetries are given by

$$
\begin{aligned}
\delta \mathcal{M} & =Y \mathcal{M}^{\prime}+2 Y^{\prime} \mathcal{M}-2 Y^{\prime \prime \prime} \\
\delta \mathcal{J} & =Y \mathcal{J}^{\prime}+2 Y^{\prime} \mathcal{J}+T \mathcal{M}^{\prime}+2 T^{\prime} \mathcal{M}+\mathcal{E} \Psi^{\prime}+3 \mathcal{E}^{\prime} \Psi-2 T^{\prime \prime \prime} \\
\delta \Psi & =Y \Psi^{\prime}+\frac{3}{2} Y^{\prime} \Psi+\frac{1}{2} \mathcal{M E}-2 \mathcal{E}^{\prime \prime}
\end{aligned}
$$

The would-be variation of the canonical generators that corresponds to the asymptotic symmetries spanned by $\lambda(T, Y, \mathcal{E})$ can be readily found in the canonical approach [52]. In the case of a Chern-Simons theory in three dimensions, they are given by [53-56]

$$
\not Q[\lambda]=-\frac{k}{2 \pi} \int\left\langle\lambda, \delta A_{\phi}\right\rangle d \phi .
$$

For the asymptotic behaviour described here, it is straightforward to verify that this expression becomes linear in the deviation of the fields with respect to the reference background, so that it can be directly integrated as

$$
Q[T, Y, \mathcal{E}]=-\frac{k}{4 \pi} \int[T \mathcal{M}+Y \mathcal{J}-2 \mathcal{E} \Psi] d \phi .
$$

Therefore, since the Poisson brackets fulfill $\delta_{\lambda_{1}} Q\left[\lambda_{2}\right]=\left\{Q\left[\lambda_{2}\right], Q\left[\lambda_{1}\right]\right\}$, the algebra of the canonical generators can be directly read from the transformation laws in (3.8). When expanded in Fourier modes,

$$
\mathcal{P}_{m}=\frac{k}{4 \pi} \int e^{i m \phi} \mathcal{M} d \phi \quad, \quad \mathcal{J}_{m}=\frac{k}{4 \pi} \int e^{i m \phi} \mathcal{J} d \phi \quad, \quad \mathcal{Q}_{m}=\frac{k}{4 \pi} \int e^{i m \phi} \Psi d \phi
$$

the Poisson brackets read explicitly

$$
\begin{aligned}
i\left\{\mathcal{P}_{m}, \mathcal{P}_{n}\right\} & =0 \\
i\left\{\mathcal{J}_{m}, \mathcal{J}_{n}\right\} & =(m-n) \mathcal{J}_{m+n}+\frac{c_{1}}{12} m^{3} \delta_{m+n, 0} \\
i\left\{\mathcal{J}_{m}, \mathcal{P}_{n}\right\} & =(m-n) \mathcal{P}_{m+n}+\frac{c_{2}}{12} m^{3} \delta_{m+n, 0} \\
i\left\{\mathcal{P}_{m}, \mathcal{Q}_{n}\right\} & =0 \\
i\left\{\mathcal{J}_{m}, \mathcal{Q}_{n}\right\} & =\left(\frac{m}{2}-n\right) \mathcal{Q}_{m+n} \\
\left\{\mathcal{Q}_{m}, \mathcal{Q}_{n}\right\} & =\mathcal{P}_{m+n}+\frac{c_{2}}{6} m^{2} \delta_{m+n, 0}
\end{aligned}
$$

where the central charges are given by $c_{1}=0$ and $c_{2}=\frac{3}{G}$. Note that the standard redefinitions $\mathcal{J}_{0} \rightarrow \mathcal{J}_{0}+\frac{c_{1}}{24}, \mathcal{P}_{0} \rightarrow \mathcal{P}_{0}+\frac{c_{2}}{24}$ change the central terms in the algebra to $\frac{c_{1}}{12} m\left(m^{2}-1\right) \delta_{m+n, 0}, \frac{c_{2}}{12} m\left(m^{2}-1\right) \delta_{m+n, 0}$ and $\frac{c_{2}}{6}\left(m^{2}-\frac{1}{4}\right)$. 
Algebra (3.11) constitutes the minimal supersymmetric extension of the $\mathrm{BMS}_{3}$ algebra with central extensions. One can furthermore show that the fields $\mathcal{M}, \mathcal{J}, \Psi$, their transformation laws (3.8) and the Poisson bracket algebra (3.11) are entirely captured by the coadjoint representation of the centrally extended super-BMS 3 group.

\section{$4 \quad$ Energy bounds and Killing spinors}

\subsection{Energy bounds from quantum superalgebra}

If the gravitino fulfills antiperiodic (Neveu-Schwarz) boundary conditions, the modes $\mathcal{Q}_{p}$ involve half-integer $p$. The wedge subalgebra is then spanned by the subset $\mathcal{P}_{m}, \mathcal{J}_{m}, \mathcal{Q}_{p}$, with $m= \pm 1,0$, and $p= \pm 1 / 2$, which corresponds to the super-Poincaré algebra. Indeed, this can be explicitly seen once the modes in (3.11) are identified with the generators in (2.3) according to $\mathcal{J}_{-1}=-\sqrt{2} J_{0}, \mathcal{J}_{1}=\sqrt{2} J_{1}, \mathcal{J}_{0}=J_{2}, \mathcal{P}_{-1}=-\sqrt{2} P_{0}, \mathcal{P}_{1}=\sqrt{2} P_{1}$, $\mathcal{P}_{0}=P_{2}-\frac{1}{8 G}, \mathcal{Q}_{1 / 2}=\sqrt{2} Q_{-}$and $\mathcal{Q}_{-1 / 2}=\sqrt{2} Q_{+}$. In the quantum theory, one can then use arguments similar to those of $[14,57,58]$ : the last of the brackets in $(3.11)$ becomes an anticommutator to lowest order in $\hbar$ and the quantum generator $\mathcal{P}_{0}$ is bounded according to

$$
\mathcal{P}_{0}=\mathcal{Q}_{1 / 2} \mathcal{Q}_{-1 / 2}+\mathcal{Q}_{-1 / 2} \mathcal{Q}_{1 / 2}-\frac{1}{8 G} \geq-\frac{1}{8 G} .
$$

In classical supergravity, the simplest solution that saturates the bound is Minkowski spacetime with $\mathcal{P}_{0}=-\frac{1}{8 G}$ and all other modes of $\mathcal{M}, \mathcal{J}, \Psi$ vanishing.

For the case of periodic (Ramond) boundary conditions for the gravitino, the modes $\mathcal{Q}_{p}$ involve integer $p$ and the bound on the quantum generator becomes

$$
\mathcal{P}_{0}=\mathcal{Q}_{0}^{2} \geq 0 \text {. }
$$

The simplest classical supergravity solution that saturates this bound is the null orbifold [59] with all modes vanishing. ${ }^{3}$

\subsection{Asymptotic Killing spinors}

Starting from transformations (3.8), one can systematically discuss the isotropy subalgebras of various solutions. A particular case of this problem is the "asymptotic Killing spinor equation", i.e., the question which asymptotic supersymmetry transformations leave purely bosonic solutions invariant,

$$
\delta_{\mathcal{E}} \Psi=-2 \mathcal{E}^{\prime \prime}+\frac{1}{2} \mathcal{M E}=0 .
$$

Asymptotic Killing spinors of solutions with constant $\mathcal{M} \neq 0$, are given by

$$
\mathcal{E}=A e^{\frac{\sqrt{\mathcal{M}}}{2} \phi}+B e^{-\frac{\sqrt{\mathcal{M}}}{2} \phi},
$$

\footnotetext{
${ }^{3}$ How to turn these arguments into a supersymmetry based proof, analogous to the one in four dimensions [60], of the positive energy theorems in classical three-dimensional general relativity [13] will be discussed elsewhere.
} 
with $A, B$ constants. They are globally well-defined provided $\mathcal{M}=-n^{2}$, with $n>0$ a strictly positive integer,

$$
\mathcal{E}=\mathcal{E}_{\frac{n}{2}} e^{i n \frac{\phi}{2}}+\mathcal{E}_{-\frac{n}{2}} e^{-i n \frac{\phi}{2}} .
$$

Solutions with $n>1$ are below the bounds (4.1) or (4.2). This singles out $n=1$, Minkowski spacetime for $\mathcal{J}=0$, in which case there are two independent antiperiodic solutions.

In the remaining case, $\mathcal{M}=0$, the solution of (4.3) is given by

$$
\mathcal{E}=\mathcal{E}_{0}+\mathcal{F}_{0} \phi
$$

with $\mathcal{E}_{0}, \mathcal{F}_{0}$ constants, which is single-valued provided $\mathcal{F}_{0}=0$. This means in particular that there is a single periodic solution for the null orbifold at $\mathcal{J}=0$.

\subsection{Exact Killing spinors of bosonic zero mode solutions}

Purely bosonic solutions $(\psi=0)$ to the field equations $(2.9)$ in the asymptotic region are described in outgoing Eddington-Finkelstein coordinates by metrics

$$
d s^{2}=\mathcal{M} d u^{2}-2 d u d r+\mathcal{N} d u d \phi+r^{2} d \phi^{2},
$$

with $\mathcal{M}, \mathcal{N}$ as in (3.7). The "zero mode solutions"

$$
\mathcal{M}=8 G M \quad, \quad \mathcal{N}=8 G J
$$

with $M, J$ constants, describe cosmological solutions for nonnegative mass $(M \geq 0)$ and arbitrary values of the angular momentum $J$, while for $-\frac{1}{8 G}<M<0$, the geometry corresponds to stationary conical defects. For $M=-\frac{1}{8 G}$, the curvature is no longer singular at the origin, but the torsion is unless $J=0$, which corresponds to Minkowski spacetime. Below this value of the mass, the geometry describes angular excesses (see, e.g., $[1,22])$.

Such solutions admit global supersymmetries when they are invariant under supersymmetry transformations of the form (2.8), provided the spinorial parameter $\epsilon$ is globally defined. The Killing spinor equation to be solved is then given by

$$
D \varepsilon=(d+\omega) \varepsilon=0,
$$

with $\omega=\frac{1}{2} \omega^{a} \Gamma_{a}$.

This equation can be solved directly through $\varepsilon=\Lambda^{-1} \varepsilon_{0}$ with $\varepsilon_{0}$ a constant spinor and $\Lambda$ the Lorentz group element associated to the flat spin connection, $\omega=\Lambda^{-1} d \Lambda$, whose form can be read off (3.2),

$$
\Lambda=\exp \left[\frac{1}{2}\left(\Gamma_{1}+\frac{1}{2} \mathcal{M} \Gamma_{0}\right) \phi\right]=\left(\begin{array}{cc}
\cosh \left(\frac{\sqrt{\mathcal{M}}}{2} \phi\right) & \sqrt{\frac{\mathcal{M}}{2}} \sinh \left(\frac{\sqrt{\mathcal{M}}}{2} \phi\right) \\
\sqrt{\frac{2}{\mathcal{M}}} \sinh \left(\frac{\sqrt{\mathcal{M}}}{2} \phi\right) & \cosh \left(\frac{\sqrt{\mathcal{M}}}{2} \phi\right)
\end{array}\right) .
$$

Alternatively, one can first solve the Killing spinor equation for the upper component. According to (3.4), this amounts to $\epsilon^{+}=-\sqrt{2} \epsilon^{-1}$. The equation for the lower component then reduces to the asymptotic Killing spinor equation (4.3). 
When suitably identifying the constants $\epsilon_{0}^{+}, \epsilon_{0}^{-}$, one finds in both cases that the Killing spinor $\varepsilon$ is globally defined provided $\mathcal{M}=-n^{2}$ with $n$ a positive integer. For $n>0$, one finds two independent Killing spinors which can be periodic (even $n$ ) or antiperiodic (odd $n$ ) given explicitly by $\epsilon=\left(-\sqrt{2} \mathcal{E}^{\prime}, \mathcal{E}\right)$, with $\mathcal{E}$ as in $(4.5)$. For $n=0$, one finds a single independent periodic solution given explicitly by $\epsilon=\left(0, \mathcal{E}_{0}\right)$.

In summary, massive cosmological solutions $(\mathcal{M}>0)$ do not admit global supersymmetries, while the massless case admits only one periodic Killing spinor. For $\mathcal{M}=-n^{2}$, the geometries possess two (the maximum number of) global supersymmetries, which includes, for $n=1$, the case of Minkowski spacetime.

Note that the geometries with $\mathcal{M}=-n^{2}, n>1$ can be interpreted as suitable unwrappings of those for $n=1$ with $n$ playing the role of the winding number. Indeed, the rescalings

$$
\phi^{\prime}=n \phi, \quad r^{\prime}=n^{-1} r, \quad u^{\prime}=n u,
$$

amount to the change $M \rightarrow n^{2} M, J \rightarrow n^{2} J$ in (4.8). As we have argued in section 4.2, these geometries actually become excluded when one insists on fulfilling the energy bounds in eqs. (4.1) and (4.2), for the periodic and antiperiodic boundary conditions, respectively.

It is worth pointing out that geometries endowed with angular deficit or excess actually possess a curvature singularity on top of the source at the origin, so that they do not fulfill the integrability condition of (4.9), i.e., $D D \varepsilon \neq 0$. Minkowski spacetime is obviously devoid of this problem, while a detailed discussion of the singularity of the null orbifold $\mathcal{M}=0=\mathcal{J}$ at $r=0$ can be found in section 2.3 of [61].

\section{$5 \quad$ Flat limit of asymptotically $\mathrm{AdS}_{3}$ supergravity}

The standard $\mathcal{N}=1$ supergravity action (2.6) can be directly recovered either from the $(1,0)$ or the $(0,1)$ AdS supergravity theory in the vanishing cosmological constant limit. However, when one deals with the asymptotic behaviour of the fields, even in the case of pure gravity, the limiting process turns out to be much more subtle [22]. In this section we show how the results obtained in section 3 can be recovered from the corresponding ones in the case of asymptotically $\mathrm{AdS}_{3}$ supergravity. Here we follow a similar strategy as the one carried out in [23] for the vanishing cosmological constant limit of higher spin gravity, which consists in finding a particularly suitable gauge choice that allows to perform the limit in a straightforward way.

\subsection{Asymptotic behaviour of minimal $\mathrm{AdS}_{3}$ supergravity, canonical generators and superconformal symmetry}

There are two inequivalent minimal locally supersymmetric extensions of General Relativity with negative cosmological constant in three spacetime dimensions, known as the $(1,0)$ and $(0,1)$ theories. Since both possess the same vanishing cosmological limit, without loss of generality we will choose the $(1,0)$ one, which can be formulated as a Chern-Simons theory whose gauge group is given by $\operatorname{OSp}(2 \mid 1) \otimes \operatorname{Sp}(2)$ [44]. The action depends on two 
independent connections $A^{+}$and $A^{-}$, for $\operatorname{OSp}(2 \mid 1)$ and $\operatorname{Sp}(2)$, respectively, and is given by

$$
I_{\mathrm{SAdS}}=I\left[A^{+}\right]-I\left[A^{-}\right],
$$

where $I[A]$ is defined in (2.4).

The asymptotic behaviour of the fields has been previously discussed in $[15,16]$. The fall-off of the fields can be written as

$$
A^{ \pm}=b_{ \pm}^{-1} a^{ \pm} b_{ \pm}+b_{ \pm}^{-1} d b_{ \pm}
$$

with $b_{ \pm}=e^{ \pm \log (r / l) L_{0}}$, and

$$
\begin{aligned}
& a^{+}=\left(L_{1}^{+}-\mathcal{L}_{+} L_{-1}^{+}+\psi Q_{+}\right) d x^{+}, \\
& a^{-}=\left(L_{-1}^{-}-\mathcal{L}_{-} L_{1}^{-}\right) d x^{-},
\end{aligned}
$$

where $x^{ \pm}=\frac{t}{l} \pm \phi$. Here the generators $L_{i}^{ \pm}$, with $i=-1,0,1$, span the left and right copies of $\operatorname{Sp}(2)$, and $Q_{\alpha}$, with $\alpha=1,-1$, correspond to the (left) fermionic generators of $\operatorname{OSp}(2 \mid 1)$. On-shell, the functions $\mathcal{L}_{ \pm}$and the Grassmann-valued $\psi$, are required to be chiral, i.e.,

$$
\partial_{\mp} \mathcal{L}_{ \pm}=0, \quad \partial_{-} \psi=0
$$

so that they depend only on $x^{+}$or $x^{-}$.

The asymptotic symmetries are given by the gauge transformations $\delta a^{ \pm}=d \lambda^{ \pm}+$ $\left[a^{ \pm}, \lambda^{ \pm}\right]$that maintain the form of (5.2), so that $\lambda^{ \pm}$are given by

$$
\lambda^{+}=\chi^{+} L_{1}-\chi^{+\prime} L_{0}+\frac{1}{2}\left(-2 \mathcal{L}_{+} \chi^{+}-\epsilon \Psi+\chi^{+\prime \prime}\right) L_{-1}+\left(\chi^{+} \Psi+\epsilon^{\prime}\right) Q_{+}+\epsilon Q_{-},
$$

and

$$
\lambda^{-}=\chi^{-} L_{-1}+\chi^{-\prime} L_{0}+\frac{1}{2}\left(-2 \mathcal{L}_{-} \chi^{-}+\chi^{-\prime \prime}\right) L_{1},
$$

which depend on three arbitrary chiral functions, fulfilling

$$
\partial_{ \pm} \chi^{\mp}=0 \quad, \quad \partial_{-} \epsilon=0 .
$$

The on-shell transformation law of the fields $\mathcal{L}_{ \pm}, \psi$ reads

$$
\begin{aligned}
\delta \mathcal{L}_{+} & =\chi^{+} \mathcal{L}_{+}^{\prime}+2 \mathcal{L}_{+} \chi_{+}^{\prime}-\frac{1}{2} \chi^{+\prime \prime \prime}+\frac{3}{2} \psi \epsilon^{\prime}+\frac{1}{2} \epsilon \psi^{\prime}, \\
\delta \psi & =-\mathcal{L}_{+} \epsilon+\epsilon^{\prime \prime}+\frac{3}{2} \psi \chi^{+\prime}+\chi^{+} \psi^{\prime}, \\
\delta \mathcal{L}_{-} & =\chi^{-} \mathcal{L}_{-}^{\prime}+2 \mathcal{L}_{-} \chi_{-}^{\prime}-\frac{1}{2} \chi^{-\prime \prime \prime} .
\end{aligned}
$$

The canonical generators associated to the asymptotic symmetries spanned by $\lambda^{+}=$ $\lambda^{+}\left(\chi^{+}, \epsilon\right)$ and $\lambda^{-}=\lambda^{-}\left(\chi^{-}\right)$, are given by

$$
\begin{aligned}
Q^{+}\left[\chi^{+}, \epsilon\right] & =-\frac{\kappa}{2 \pi} \int\left[\chi^{+} \mathcal{L}_{+}-\epsilon \psi\right] d \phi, \\
Q^{-}\left[\chi^{-}\right] & =-\frac{\kappa}{2 \pi} \int\left[\chi^{-} \mathcal{L}_{-}\right] d \phi,
\end{aligned}
$$


where $\kappa:=l k$, which by virtue of (5.5), can be readily shown to fulfill the (super) Virasoro algebra. Expanding in Fourier modes $\mathcal{L}_{m}^{ \pm}=\frac{k l}{4 \pi} \int \mathcal{L}_{ \pm} e^{ \pm i m \phi} d \phi$ and $\mathcal{Q}_{m}=\frac{k l}{4 \pi} \int \psi e^{i m \phi} d \phi$, the nonvanishing Poisson brackets read

$$
\begin{aligned}
i\left\{\mathcal{L}_{m}^{ \pm}, \mathcal{L}_{n}^{ \pm}\right\} & =(m-n) \mathcal{L}_{n+m}^{ \pm}+\frac{c}{12} m^{3} \delta_{m+n, 0}, \\
i\left\{\mathcal{L}_{m}^{+}, \mathcal{Q}_{n}^{+}\right\} & =\left(\frac{m}{2}-n\right) \mathcal{Q}_{m+n}^{+} \\
\left\{\mathcal{Q}_{m}^{+}, \mathcal{Q}_{n}^{+}\right\} & =2 \mathcal{L}_{m+n}^{+}+\frac{c}{3} m^{2} \delta_{m+n, 0}
\end{aligned}
$$

where the central charge is given by $c=\frac{3 l}{2 G}$.

\subsection{Vanishing cosmological constant limit}

In order to recover the results of section 3 from the ones described in the previous subsection once the vanishing cosmological constant limit is taken, it turns out to be useful to express the asymptotic behaviour of the gauge fields of the $(1,0)$ AdS supergravity theory in a different gauge. We then choose different group elements $g_{ \pm}$, so that the fall-off of the connections now read

$$
A^{ \pm}=g_{ \pm}^{-1} a^{ \pm} g_{ \pm}+g_{ \pm}^{-1} d g_{ \pm}
$$

where $a^{ \pm}$are given by (5.2), and

$$
\begin{aligned}
& g_{+}=b_{+} e^{-\log \left(\frac{r}{l}\right) L_{0}} e^{\frac{r}{2 l} L_{-1}}, \\
& g_{-}=b_{-} e^{-\log \left(\frac{r}{4 l}\right) L_{0}} e^{\frac{r}{2 l} L_{-1}} e^{\frac{2 l}{r} L_{1}} .
\end{aligned}
$$

In this gauge, the asymptotic form of the super-AdS gauge field is explicitly given by

$$
\begin{aligned}
& A^{+}=\frac{r}{l} d x^{+} L_{0}^{+}+\frac{1}{2}\left[\frac{d r}{l}+\left(\frac{r^{2}}{2 l^{2}}-2 \mathcal{L}_{+}\right) d x^{+}\right] L_{-1}^{+}+d x^{+} L_{1}^{+}+\psi^{+} Q_{+} d x^{+}, \\
& A^{-}=\frac{r}{l} d x^{-} L_{0}^{-}-\frac{1}{2}\left[\frac{d r}{l}+\left(\frac{r^{2}}{2 l^{2}}-2 \mathcal{L}_{-}\right) d x^{-}\right] L_{-1}^{-}-d x^{-} L_{1}^{-}
\end{aligned}
$$

It is now convenient to make the change $t=u$ and to perform the change of basis

$$
L_{-1}^{( \pm)}=-2 J_{0}^{ \pm}, \quad L_{0}^{ \pm}=J_{2}^{ \pm}, \quad L_{1}^{( \pm)}=J_{1}^{ \pm}, \quad Q_{+}=\frac{1}{2^{1 / 4}} \tilde{Q}_{+},
$$

followed by

$$
J_{a}^{ \pm}=\frac{J_{a} \pm l P_{a}}{2}, \quad Q_{+}=\sqrt{l} \tilde{Q}_{+},
$$

so that the full gauge field reads

$$
\begin{aligned}
A= & \left(-d r+\frac{\mathcal{M}}{2} d u+\frac{\mathcal{N}}{2} d \phi-\frac{r^{2}}{2 l^{2}} d u\right) P_{0}+d u P_{1}+r d \phi P_{2} \\
& +\left(\frac{\mathcal{M}}{2} d \phi+\frac{\mathcal{N}}{2 l^{2}} d u-\frac{r^{2}}{2 l^{2}} d \phi\right) J_{0} \\
& +d \phi J_{1}+\frac{r}{l^{2}} d u J_{2}+\frac{\Psi}{2^{1 / 4}} \tilde{Q}_{+} d \phi+\frac{1}{l} \frac{\Psi}{2^{1 / 4}} \tilde{Q}_{+} d u,
\end{aligned}
$$


where the arbitrary functions $\mathcal{L}_{ \pm}, \psi$ have been redefined according to

$$
\mathcal{M}=\left(\mathcal{L}_{+}+\mathcal{L}_{-}\right), \quad \mathcal{N}=l\left(\mathcal{L}_{+}-\mathcal{L}_{-}\right), \quad \Psi=\sqrt{l} \psi
$$

The chirality conditions (5.3) now read

$$
\partial_{u} \mathcal{M}=\frac{1}{l^{2}} \partial_{\phi} \mathcal{N} \quad, \quad \partial_{u} \mathcal{N}=\partial_{\phi} \mathcal{M} \quad, \quad \partial_{u} \Psi=\frac{1}{l} \partial_{\phi} \Psi .
$$

The vanishing cosmological constant limit can now be directly performed in a transparent way. Indeed, for the full gauge field $A=A^{+}+A^{-}$, one just takes $l \rightarrow \infty$, so that it reduces to

$$
A=\left(-d r+\frac{\mathcal{M}}{2} d u+\frac{\mathcal{N}}{2} d \phi\right) P_{0}+d u P_{1}+r d \phi P_{2}+\frac{\mathcal{M}}{2} d \phi J_{0}+d \phi J_{1}+\frac{\Psi}{2^{1 / 4}} Q_{+} d \phi,
$$

which coincides with the asymptotic form of the connection in the asymptotically flat case, eqs. (3.1), (3.2). Analogously, in the limit, the chirality conditions (5.15) take the flat space form (3.6), whose solution is given by (3.7).

It is simple to verify that the expression for the global charges for the gauge choice (5.9) remains the same as in the gauge (5.1) and is still given by (5.6). After making use of the redefinition for the fields in (5.14), they acquire the form

$$
Q[f, h, \mathcal{E}]=-\frac{k}{4 \pi} \int d \phi(f \mathcal{M}+h \mathcal{N}-2 \mathcal{E} \Psi),
$$

where the parameters that characterize the asymptotic symmetries have been conveniently redefined as

$$
f=l\left(\chi^{+}+\chi^{-}\right), \quad h=\chi^{+}-\chi^{-}, \quad \mathcal{E}=\sqrt{l} \varepsilon .
$$

The chirality conditions (5.4) on the gauge parameters then read

$$
\partial_{u} f=\partial_{\phi} h, \quad \partial_{u} h=\frac{1}{l^{2}} \partial_{\phi} f, \quad \partial_{u} \mathcal{E}=\frac{1}{l} \partial_{\phi} \mathcal{E},
$$

and, in the limit $l \rightarrow \infty$, they imply that

$$
h=Y(\phi), \quad f=T(\phi)+u Y^{\prime}, \quad \mathcal{E}=\mathcal{E}(\phi),
$$

and hence, by virtue of (3.7), the global charges (5.16) reduce to the ones of the asymptotically flat case given in (3.10).

As explained in section 5.1, the canonical generators of (1,0) AdS supergravity satisfy the centrally-extended superconformal algebra in two dimensions given by (5.7). In order to take the flat limit, it is useful to change the basis according to

$$
\mathcal{P}_{m} \equiv \frac{1}{l}\left(\mathcal{L}_{m}^{+}+\mathcal{L}_{-m}^{-}\right), \quad \mathcal{J}_{m} \equiv \mathcal{L}_{m}^{+}-\mathcal{L}_{-m}^{-},
$$

as well as rescaling the supercharges as

$$
\mathcal{Q}_{m} \equiv \frac{1}{\sqrt{l}} \mathcal{Q}_{m}^{+}
$$

After this has been done, in the limit $l \rightarrow \infty$, algebra (5.7) readily reduces to the minimal supersymmetric extension of the $\mathrm{BMS}_{3}$ algebra (3.11), where the central charges are given by $l c_{1}=c^{+}-c^{-}$and $l c_{2}=c^{+}+c^{-}$. In particular, it also follows that the bounds for the generators that are obtained from the superconformal algebra, reduce to the ones in eqs. (4.1) and (4.2) in the limit of vanishing cosmological constant. 


\section{Asymptotic structure of $\mathcal{N}=1$ "reloaded" flat supergravity}

The locally supersymmetric extension of the most general three-dimensional gravity theory that leads to first order field equations for the dreibein and the spin connection has been constructed in [49]. It includes additional parity-odd terms as compared with the standard theory. In the vanishing cosmological constant limit, the action with $\mathcal{N}=1$ supersymmetry is given by

$$
\begin{aligned}
I_{(\mu, \gamma)}=\frac{k}{4 \pi} \int 2(1+\mu \gamma) R^{a} e_{a}+\gamma^{2}(1+\mu & \left.\frac{\gamma}{3}\right) \epsilon_{a b c} e^{a} e^{b} e^{c}+\mu L(\omega) \\
& +\gamma(2+\mu \gamma) T^{a} e_{a}-\bar{\psi}\left(D+\frac{\gamma}{2} e^{a} \Gamma_{a}\right) \psi,
\end{aligned}
$$

where $L(\omega)=\omega^{a} d \omega_{a}+\frac{1}{3} \epsilon_{a b c} \omega^{a} \omega^{b} \omega^{c}$ is the Lorentz-Chern-Simons form. This action is invariant, up to a surface term, under the following local supersymmetry transformations

$$
\delta e^{a}=\frac{1}{2} \bar{\epsilon} \Gamma^{a} \psi, \quad \delta \omega^{a}=\frac{1}{2} \gamma \bar{\psi} \Gamma^{a} \epsilon, \quad \delta \psi=D \epsilon+\frac{1}{2} \gamma e^{a} \Gamma_{a} \epsilon .
$$

Note that in the case of $\mu=\gamma=0$, the action (6.1) and the supersymmetry transformations (6.2) reduce to the standard ones, given by (2.6) and (2.8), respectively.

Remarkably, in spite of the presence of a volume term in (6.1), the theory can also be formulated in terms of a Chern-Simons action for the super-Poincaré group. This can be seen as follows. In terms of the shifted spin connection $\hat{\omega}^{a}:=\omega^{a}+\gamma e^{a}$, action (6.1) reads

$$
I_{(\mu, \gamma)}=\frac{k}{4 \pi} \int 2 \hat{R}^{a} e_{a}+\mu L(\hat{\omega})-\psi_{\alpha} \hat{D} \psi^{\alpha},
$$

where $\hat{D}, \hat{R}^{a}$, and $L(\hat{\omega})$ stand for the covariant derivative, the curvature two-form, and the Lorentz-Chern-Simons form constructed out from $\hat{\omega}^{a}$, respectively. Hence, up to a boundary term, the action can be written as

$$
I[A]=\frac{k}{4 \pi} \int\left\langle A, d A+\frac{2}{3} A^{2}\right\rangle,
$$

where now the gauge field is given by

$$
A=e^{a} P_{a}+\hat{\omega}^{a} J_{a}+\psi^{\alpha} Q_{\alpha},
$$

and the nonvanishing components of the invariant nondegenerate bilinear form read

$$
\left\langle P_{a}, J_{b}\right\rangle=\eta_{a b}, \quad\left\langle J_{a}, J_{b}\right\rangle=\mu \eta_{a b}, \quad\left\langle Q_{\alpha}, Q_{\beta}\right\rangle=C_{\alpha \beta},
$$

so that it reduces to the standard bracket in (2.5) in the case of $\mu=0$.

The asymptotic behaviour of the gauge fields in this case is then proposed to be exactly of the same form as in eqs. (3.1), (3.2), which by virtue of (6.5), amounts just to modify the fall-off of the spin connection $\omega^{a}$ in the asymptotic region. This has to be so because the field equations now imply a nonvanishing torsion even in vacuum. 
Therefore, the asymptotic symmetries are spanned by the same Lie-algebra valued parameter $\lambda=\lambda(T, Y, \mathcal{E})$ as in section 3 but, since the invariant form has been modified according to (6.6), the global charges acquire a correction, so that they now read

$$
\mathcal{Q}[T, Y, \mathcal{E}]=-\frac{k}{4 \pi} \int[T \mathcal{M}+Y(\mathcal{J}+\mu \mathcal{M})-2 \mathcal{E} \Psi] d \phi .
$$

Consequently, once expanded in modes, the Poisson bracket algebra of the canonical generators are given by the minimal supersymmetric extension of the $\mathrm{BMS}_{3}$ algebra (3.11), but with an additional central charge,

$$
c_{1}=\mu \frac{3}{G}, \quad c_{2}=\frac{3}{G} .
$$

\section{Acknowledgments}

We thank M. Bañados, C. Bunster, O. Fuentealba, H. González, M. Henneaux, P.-H. Lambert, C. Martínez, A. Pérez, D. Tempo, and C. Troessaert for enlightening discussions. J.M. and R.T. wish to thank the Physique théorique et mathématique group of the Université Libre de Bruxelles, and the International Solvay Institutes for the warm hospitality. J.M. also thanks Conicyt for financial support. This work is partially funded by the Fondecyt grants $N^{\circ} 1130658,1121031$. The Centro de Estudios Científicos (CECs) is funded by the Chilean Government through the Centers of Excellence Base Financing Program of Conicyt. G.B. is research director of the Fund for Scientific Research-FNRS Belgium, while L.D. is a research fellow of the "Fonds pour la Formation à la Recherche dans l'Industrie et dans l'Agriculture"-FRIA Belgium. Their work is supported in part by IISN-Belgium and by "Communauté française de Belgique - Actions de Recherche Concerteées".

\section{A Conventions}

Our conventions are such that the Levi-Civita symbol fulfills $\epsilon_{012}=1$, and the tangent space metric $\eta_{a b}$, with $a=0,1,2$, is off-diagonal, given by

$$
\eta_{a b}=\left(\begin{array}{lll}
0 & 1 & 0 \\
1 & 0 & 0 \\
0 & 0 & 1
\end{array}\right)
$$

The three-dimensional $\Gamma$-matrices satisfy the Clifford algebra $\left\{\Gamma_{a}, \Gamma_{b}\right\}=2 \eta_{a b}$, and are chosen as

$$
\Gamma_{0}=\frac{1}{\sqrt{2}}\left(\sigma_{1}+i \sigma_{2}\right), \quad \Gamma_{1}=\frac{1}{\sqrt{2}}\left(\sigma_{1}-i \sigma_{2}\right), \quad \Gamma_{2}=\sigma_{3},
$$

where the $\sigma$ 's stand for the Pauli matrices

$$
\sigma_{1}=\left(\begin{array}{ll}
0 & 1 \\
1 & 0
\end{array}\right), \quad \sigma_{2}=\left(\begin{array}{cc}
0 & -i \\
i & 0
\end{array}\right), \quad \sigma_{3}=\left(\begin{array}{cc}
1 & 0 \\
0 & -1
\end{array}\right) .
$$

As a consequence, they satisfy

$$
\Gamma_{a} \Gamma_{b}=\epsilon_{a b c} \Gamma^{c}+\eta_{a b} \mathbf{1}, \quad \Gamma^{a \alpha}{ }_{\beta} \Gamma_{a \delta}^{\gamma}=2 \delta_{\delta}^{\alpha} \delta_{\beta}^{\gamma}-\delta_{\beta}^{\alpha} \delta_{\delta}^{\gamma},
$$

where tangent space indices are lowered and raised with $\eta_{a b}$ and its inverse. 
For a spinor $\psi^{\alpha}$, with $\alpha=+1,-1$, we define the Majorana conjugate as $\bar{\psi}_{\alpha}=C_{\alpha \beta} \psi^{\beta}$, where the charge conjugation matrix is given by $C=i \sigma_{2}$, that is

$$
C_{\alpha \beta}=\varepsilon_{\alpha \beta}=C^{\alpha \beta}=\left(\begin{array}{cc}
0 & 1 \\
-1 & 0
\end{array}\right),
$$

which satisfies $C^{T}=-C$ and $C \Gamma_{a} C^{-1}=-\left(\Gamma_{a}\right)^{T}$. In particular, this implies $\overline{\Lambda^{-1} \psi}=\bar{\psi} \Lambda$ if $\Lambda \in \mathrm{SL}(2, \mathbf{R})$.

Open Access. This article is distributed under the terms of the Creative Commons Attribution License (CC-BY 4.0), which permits any use, distribution and reproduction in any medium, provided the original author(s) and source are credited.

\section{References}

[1] S. Deser, R. Jackiw and G. 't Hooft, Three-dimensional Einstein gravity: dynamics of flat space, Annals Phys. 152 (1984) 220 [InSPIRE].

[2] S. Deser and R. Jackiw, Three-dimensional cosmological gravity: dynamics of constant curvature, Annals Phys. 153 (1984) 405 [INSPIRE].

[3] M. Henneaux, Energy momentum, angular momentum, and supercharge in $2+1$ supergravity, Phys. Rev. D 29 (1984) 2766 [INSPIRE].

[4] S. Deser, Breakdown of asymptotic Poincaré invariance in $D=3$ Einstein gravity, Class. Quant. Grav. 2 (1985) 489 [inSPIRE].

[5] E. Witten, Is supersymmetry really broken?, Int. J. Mod. Phys. A 10 (1995) 1247 [hep-th/9409111] [INSPIRE].

[6] J.D. Brown and M. Henneaux, Central charges in the canonical realization of asymptotic symmetries: an example from three-dimensional gravity, Commun. Math. Phys. 104 (1986) 207 [inSPIRE].

[7] M. Bañados, C. Teitelboim and J. Zanelli, The black hole in three-dimensional space-time, Phys. Rev. Lett. 69 (1992) 1849 [hep-th/9204099] [INSPIRE].

[8] M. Bañados, M. Henneaux, C. Teitelboim and J. Zanelli, Geometry of the $(2+1)$ black hole, Phys. Rev. D 48 (1993) 1506 [gr-qc/9302012] [INSPIRE].

[9] J. Navarro-Salas and P. Navarro, Virasoro orbits, AdS $S_{3}$ quantum gravity and entropy, JHEP 05 (1999) 009 [hep-th/9903248] [INSPIRE].

[10] T. Nakatsu, H. Umetsu and N. Yokoi, Three-dimensional black holes and Liouville field theory, Prog. Theor. Phys. 102 (1999) 867 [hep-th/9903259] [INSPIRE].

[11] A. Maloney and E. Witten, Quantum gravity partition functions in three dimensions, JHEP 02 (2010) 029 [arXiv:0712.0155] [INSPIRE].

[12] A. Garbarz and M. Leston, Classification of boundary gravitons in $A d S_{3}$ gravity, JHEP 05 (2014) 141 [arXiv: 1403.3367] [INSPIRE].

[13] G. Barnich and B. Oblak, Holographic positive energy theorems in three-dimensional gravity, Class. Quant. Grav. 31 (2014) 152001 [arXiv:1403.3835] [INSPIRE]. 
[14] O. Coussaert and M. Henneaux, Supersymmetry of the $(2+1)$ black holes, Phys. Rev. Lett. 72 (1994) 183 [hep-th/9310194] [INSPIRE].

[15] M. Bañados, K. Bautier, O. Coussaert, M. Henneaux and M. Ortiz, Anti-de Sitter/CFT correspondence in three-dimensional supergravity, Phys. Rev. D 58 (1998) 085020 [hep-th/9805165] [INSPIRE].

[16] M. Henneaux, L. Maoz and A. Schwimmer, Asymptotic dynamics and asymptotic symmetries of three-dimensional extended AdS supergravity, Annals Phys. 282 (2000) 31 [hep-th/9910013] [INSPIRE].

[17] A. Ashtekar, J. Bicak and B.G. Schmidt, Asymptotic structure of symmetry reduced general relativity, Phys. Rev. D 55 (1997) 669 [gr-qc/9608042] [INSPIRE].

[18] G. Barnich and G. Compere, Classical central extension for asymptotic symmetries at null infinity in three spacetime dimensions, Class. Quant. Grav. 24 (2007) F15 [gr-qc/0610130] [INSPIRE].

[19] G. Barnich and C. Troessaert, Aspects of the BMS/CFT correspondence, JHEP 05 (2010) 062 [arXiv:1001.1541] [INSPIRE].

[20] H.R. Afshar, Flat/AdS boundary conditions in three dimensional conformal gravity, JHEP 10 (2013) 027 [arXiv:1307.4855] [INSPIRE].

[21] A. Bagchi and R. Fareghbal, BMS/GCA redux: towards flatspace holography from non-relativistic symmetries, JHEP 10 (2012) 092 [arXiv:1203.5795] [INSPIRE].

[22] G. Barnich, A. Gomberoff and H.A. Gonzalez, The flat limit of three dimensional asymptotically Anti-de Sitter spacetimes, Phys. Rev. D 86 (2012) 024020 [arXiv: 1204.3288] [INSPIRE].

[23] H.A. Gonzalez, J. Matulich, M. Pino and R. Troncoso, Asymptotically flat spacetimes in three-dimensional higher spin gravity, JHEP 09 (2013) 016 [arXiv:1307.5651] [INSPIRE].

[24] R.N. Caldeira Costa, Aspects of the zero $\Lambda$ limit in the AdS/CFT correspondence, arXiv: 1311.7339 [INSPIRE].

[25] R. Fareghbal and A. Naseh, Flat-space energy-momentum tensor from BMS/GCA correspondence, JHEP 03 (2014) 005 [arXiv: 1312.2109] [INSPIRE].

[26] C. Krishnan, A. Raju and S. Roy, A Grassmann path from $A d S_{3}$ to flat space, JHEP 03 (2014) 036 [arXiv: 1312.2941] [INSPIRE].

[27] L. Cornalba and M.S. Costa, A new cosmological scenario in string theory, Phys. Rev. D 66 (2002) 066001 [hep-th/0203031] [INSPIRE].

[28] L. Cornalba and M.S. Costa, Time dependent orbifolds and string cosmology, Fortsch. Phys. 52 (2004) 145 [hep-th/0310099] [INSPIRE].

[29] G. Barnich, Entropy of three-dimensional asymptotically flat cosmological solutions, JHEP 10 (2012) 095 [arXiv: 1208.4371] [INSPIRE].

[30] A. Bagchi, S. Detournay, R. Fareghbal and J. Simon, Holography of 3D flat cosmological horizons, Phys. Rev. Lett. 110 (2013) 141302 [arXiv:1208.4372] [INSPIRE].

[31] A. Strominger, Black hole entropy from near horizon microstates, JHEP 02 (1998) 009 [hep-th/9712251] [INSPIRE]. 
[32] O. Coussaert, M. Henneaux and P. van Driel, The asymptotic dynamics of three-dimensional Einstein gravity with a negative cosmological constant, Class. Quant. Grav. 12 (1995) 2961 [gr-qc/9506019] [INSPIRE].

[33] G. Barnich, A. Gomberoff and H.A. González, Three-dimensional Bondi-Metzner-Sachs invariant two-dimensional field theories as the flat limit of Liouville theory, Phys. Rev. D 87 (2013) 124032 [arXiv:1210.0731] [InSPIRE].

[34] P. Salomonson, B.S. Skagerstam and A. Stern, Iso $(2,1)$ chiral models and quantum gravity in (2+1)-dimensions, Nucl. Phys. B 347 (1990) 769 [INSPIRE].

[35] G. Barnich and H.A. Gonzalez, Dual dynamics of three dimensional asymptotically flat Einstein gravity at null infinity, JHEP 05 (2013) 016 [arXiv:1303.1075] [INSPIRE].

[36] G. Barnich and B. Oblak, to appear.

[37] A. Bagchi and I. Mandal, Supersymmetric extension of galilean conformal algebras, Phys. Rev. D 80 (2009) 086011 [arXiv:0905.0580] [INSPIRE].

[38] I. Mandal, Supersymmetric extension of GCA in 2d, JHEP 11 (2010) 018 [arXiv: 1003.0209] [INSPIRE].

[39] M. Sakaguchi, Super galilean conformal algebra in AdS/CFT, J. Math. Phys. 51 (2010) 042301 [arXiv:0905.0188] [inSPIRE].

[40] J.A. de Azcarraga and J. Lukierski, Galilean superconformal symmetries, Phys. Lett. B 678 (2009) 411 [arXiv:0905.0141] [InSPIRE].

[41] S. Deser and J.H. Kay, Topologically massive supergravity, Phys. Lett. B 120 (1983) 97 [INSPIRE].

[42] S. Deser, Quantum theory of gravity: essays in honor of the 60th birthday of Bryce S. DeWitt, Adam Hilger Ltd., U.S.A. (1984).

[43] N. Marcus and J.H. Schwarz, Three-dimensional supergravity theories, Nucl. Phys. B 228 (1983) 145 [inSPIRE].

[44] A. Achucarro and P.K. Townsend, A Chern-Simons action for three-dimensional Anti-de Sitter supergravity theories, Phys. Lett. B 180 (1986) 89 [InSPIRE].

[45] A. Achucarro and P.K. Townsend, Extended supergravities in $d=(2+1)$ as Chern-Simons theories, Phys. Lett. B 229 (1989) 383 [INSPIRE].

[46] H. Nishino and S.J. Gates Jr., Chern-Simons theories with supersymmetries in three-dimensions, Int. J. Mod. Phys. A 8 (1993) 3371 [INSPIRE].

[47] P.S. Howe, J.M. Izquierdo, G. Papadopoulos and P.K. Townsend, New supergravities with central charges and Killing spinors in (2+1)-dimensions, Nucl. Phys. B 467 (1996) 183 [hep-th/9505032] [INSPIRE].

[48] M. Bañados, R. Troncoso and J. Zanelli, Higher dimensional Chern-Simons supergravity, Phys. Rev. D 54 (1996) 2605 [gr-qc/9601003] [INSPIRE].

[49] A. Giacomini, R. Troncoso and S. Willison, Three-dimensional supergravity reloaded, Class. Quant. Grav. 24 (2007) 2845 [hep-th/0610077] [INSPIRE].

[50] R.K. Gupta and A. Sen, Consistent truncation to three dimensional (Super-)gravity, JHEP 03 (2008) 015 [arXiv:0710.4177] [InSPIRE]. 
[51] O. Fierro, F. Izaurieta, P. Salgado and O. Valdivia, $(2+1)$-dimensional supergravity invariant under the AdS-Lorentz superalgebra, arXiv:1401.3697 [INSPIRE].

[52] T. Regge and C. Teitelboim, Role of surface integrals in the hamiltonian formulation of general relativity, Annals Phys. 88 (1974) 286 [INSPIRE].

[53] A.P. Balachandran, G. Bimonte, K.S. Gupta and A. Stern, Conformal edge currents in Chern-Simons theories, Int. J. Mod. Phys. A 7 (1992) 4655 [hep-th/9110072] [INSPIRE].

[54] M. Bañados, Global charges in Chern-Simons field theory and the $(2+1)$ black hole, Phys. Rev. D 52 (1996) 5816 [hep-th/9405171] [INSPIRE].

[55] S. Carlip, Conformal field theory, $(2+1)$-dimensional gravity and the BTZ black hole, Class. Quant. Grav. 22 (2005) R85 [gr-qc/0503022] [INSPIRE].

[56] A. Perez, D. Tempo and R. Troncoso, Brief review on higher spin black holes, arXiv:1402.1465 [INSPIRE].

[57] S. Deser and C. Teitelboim, Supergravity has positive energy, Phys. Rev. Lett. 39 (1977) 249 [INSPIRE].

[58] L.F. Abbott and S. Deser, Stability of gravity with a cosmological constant, Nucl. Phys. B 195 (1982) 76 [inSPIRE].

[59] G.T. Horowitz and A.R. Steif, Singular string solutions with nonsingular initial data, Phys. Lett. B 258 (1991) 91 [INSPIRE].

[60] E. Witten, A simple proof of the positive energy theorem, Commun. Math. Phys. 80 (1981) 381 [inSPIRE].

[61] H. Liu, G.W. Moore and N. Seiberg, Strings in a time dependent orbifold, JHEP 06 (2002) 045 [hep-th/0204168] [INSPIRE]. 